\title{
Design and Study of a Dispenser for the Introduction of the Main Batch of Mineral Fertilizers
}

\author{
Dzhadyger ESKHOZHIN, Sayakhat NUKESHEV*, Ziyada ZHAKSYLYKOVA, \\ Kairat ESKHOZHIN, Aigul BALABEKOVA \\ S. Seifullin Kazakh Agrotechnical University, Astana, Pobedy 62, 010011, Astana, Kazakhstan, \\ *E-mail: sayakhat.nuk@gmail.com \\ cross $^{\text {ref }}$ http://dx.doi.org/10.5755/j01.mech.24.3.17731
}

\section{Introduction}

In Northern and Central Kazakhstan, soil is notable for its deficit of phosphorus and nitrogen. Studies have shown that $50 \%$ of the cultivated area in this region are exposed to phosphorus shortage [1]. An agrochemical analysis showed that deep and root habitable layers are exposed to nitrogen element shortage. Therefore, the introduction of phosphorus and nitrogen fertilizers for spring crops in said regions is especially effective. Studies confirmed that this measure alone is capable of providing additional two-three hundredweight per hectare of crop yield [2].

In terms of its introduction period and technique, the introduction of mineral fertilizers is divided into presowing broadcast - main, at-sowing - simultaneously with the sowing of seeds, and post-sowing - mostly for cleancultivated crops during inter-row cultivation [3].

Pre-sowing broadcast introduction is used in case of traditional moldbroad plowing. However, due to the general exposure of Central and Northern Kazakhstan soil to wind erosion, moldbroad plowing is impossible in this region. Subsurface cultivation has become widespread here. Therefore, pre-sowing introduction of mineral fertilizers is carried out using fertilizer-dispenser cultivators based on blade machines [4].

The problem of soil wind erosion is not limited to the Republic of Kazakhstan. Although this problem is generally associated with subtropical, arid or semiarid regions, even in Europe, wind erosion is a well-known threat in many parts of Northern Germany, Eastern Netherlands, Eastern England, Southeastern Europe, and the Iberian Peninsula [Behbahani, Ali Mohammadian, et al. "Nutrient and dust enrichment in Danish wind erosion sediments for different tillage directions $[5,6,7]$.

The main machines used in the region under consideration (Central and Northern Kazakhstan) are fertilizer dispensers GUN-4, PN-8-5, and RUN-4, the operating devices whereof are blade hoes, raking props or chisel plows, and a stud-roller feed. Laboratory and field experiments showed that the introduction unevenness when using these machines reached $48-50 \%$, which is why they were not in demand $[8,9]$.

Seeding machines with at-seeding introduction of fertilizers use pin-roller feeds. Studies found that these machines failed to meet agriculture requirements to evenness and stability of introduction and did not provide for an increased (up to $400 \mathrm{~kg}$ per hectare) batch [10].

The main qualitative indicator of the introduction of mineral fertilizers into the soil is their even distribution in the soil. Results of observations in 20 oblasts of Russia showed that uneven introduction of fertilizers reduced the crop yield of wheat and barley by $13 \%$ on average. Field experiments in Germany showed that increasing the evenness of nitric fertilizer distribution from $15 \%$ to $30 \%$ increased the crop yield of spring wheat from $21.8 \%$ to $31.2 \%$ and that of spring barley from $10.1 \%$ to $17.1 \%$ [11, 12].

Based on the above, one can conclude that the problem of even batching and introduction of mineral fertilizers is especially relevant for agricultural land exposed to subsurface cultivation. This problem can be solved by designing a seeding device that is capable of evenly dispensing a larger batch.

The belt-pin mechanism was chosen among various options for achieving an even introduction of mineral fertilizers, since this option was the most realizable one, required the least changes to existing and currently operating agricultural machinery, and allowed for a significant variation of the standard introduced batch (including its increase), while the lack of portion feed provides for an even introduction. Therefore, the design and rationalization of constructive and technological parameters of the belt-pin dispenser became the main goals of this study. In order to rationalize the engineering decisions, the pilot rig was manufactured with various versions of the belt-pin mechanism; all versions underwent comprehensive trials; the analysis of results allowed choosing the optimal configuration and the optimal operating conditions of the proposed belt-pin dispenser.

\section{Materials and methods}

As a result of analytical and experimental work, the S. Seifullin Kazakh Agrotechnical University designed, manufactured, and studied a belt-pin mineral fertilizer dispenser [14]. It consists of a fertilizer box 1 with a spade agitator 2. Through a flap, the fertilizer enters the stabilization chamber 4 with the agitator 5 located in its cross section. The conveyor belt 6 removes the fertilizer, which is partially distributed across its width, from under the stabilization chamber. The spring equalizer 7 does not let lump particles through and facilitates their breakage and additional redistribution across the belt width. Pins 8 are located on the surface of the conveyor belt. They can be cylindrical, semi-cylindrical, rhomboid, rectangular, semispherical, etc. Their shapes and placement patterns are set based on technological requirements. The material on the conveyor belt with pins is distributed evenly across the width of the belt due to the pins' longitudinal vibrations. From the conveyor belt, the fertilizer enters the receiving box 9, the width whereof 
is divided into even segments $\mathrm{A}, \mathrm{B}, \mathrm{C} \ldots$, which are connected to shares.

The machine can provide for an increased introduction standard, while the lack of portion feed provides for an even distribution of the fertilizer. Preliminary laboratory experiments confirmed the potential of the chosen construction.

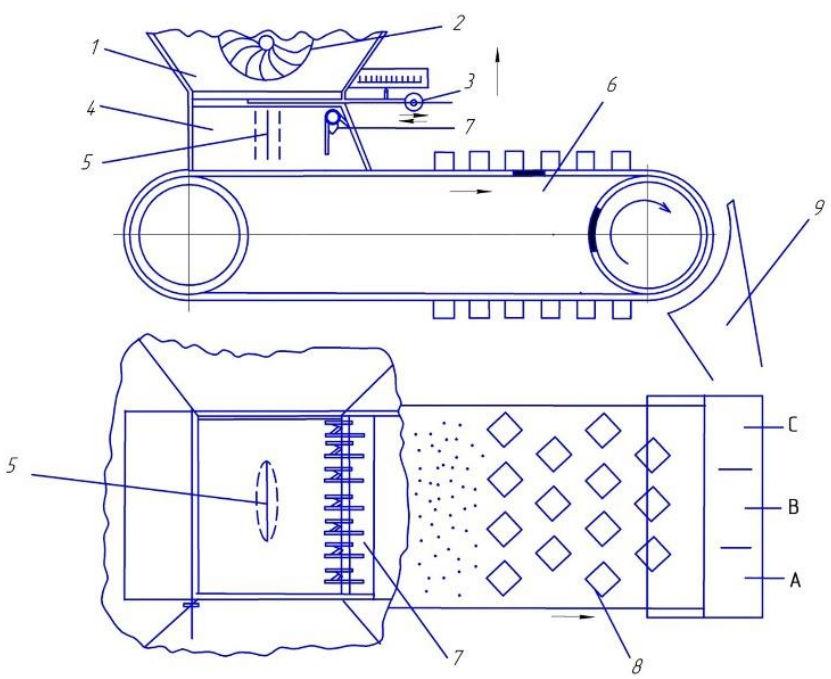

Fig. 1 Belt-pin dispenser for mineral fertilizers

The intended purpose of the belt-pin dispenser is to transport mineral fertilizers and evenly distribute the material across its surface, which is characterized by a probable identical material weight on each of its square centimeters. This work is done by pins that are located on the surface of the conveyor belt and vibrate independently together with the belt. In order to do this properly, the pins should be located evenly in the longitudinal and transverse directions; each pin should have its own trace, if possible.

In order to meet the above requirements to pin placement, it is expedient to take into consideration the geometric method of S.Ya. Rozmann, which is used to design spike-tooth harrows [15].

It is necessary to highlight a rectangular segment of the conveyor belt with $B$ width and $H$ height. A straight pin line $A D$ is drawn at angle $\alpha$ towards the base and divided into equal segments with d length. It is necessary to draw a non-isosceles triangle on the $A B$ segment, so that segments $A 1>1 C$ or $A 1<1 C$. The fact of the matter is that if $\triangle A B C$ is isosceles, then rhombus $B B_{1} C_{1} C$ is also isosceles. In this case, the pins that are located at the apexes of top and bottom rhombuses will follow each other. Therefore, it is necessary to form non-isosceles rhombuses. This is possible of triangles $\mathrm{ABC}$ are non-isosceles.

Obviously:

$$
A D=1=d N
$$

where: $d$ is the distance between pins on the $A D$ line; $N$ is the number of pins on the $A D$ line.

The tilt angle of the $A D$ line in respect to the $A A_{2}$ base is:

$$
\frac{A A_{1}}{A D}=\frac{t}{1}=\cos \alpha
$$

where: $t=A A_{1}-$ the step of the pin line.

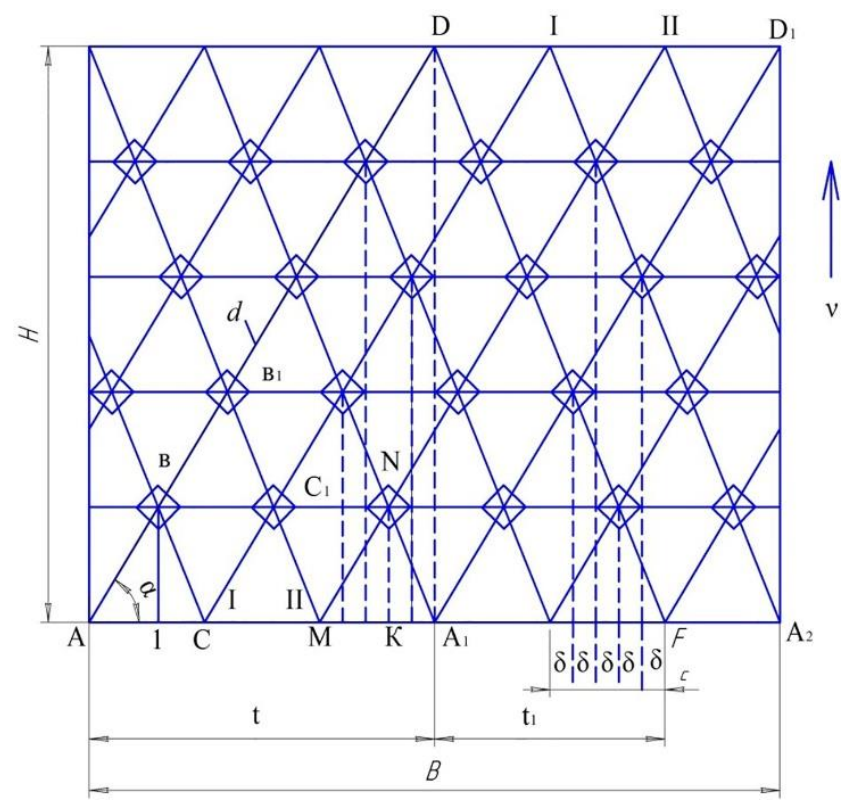

Fig. 2 Pin placement on the conveyor belt surface

In the $A A_{1}$ step, one can draw additional pin lines (I-I) (II-II) $A D$. In order to determine their number, it is necessary to divide the pin line step by the base of the ABC triangle:

$$
\begin{aligned}
& A C=c, \\
& Z_{p t}=\frac{t}{c} .
\end{aligned}
$$

The number of pin traces in step $t$ can be determined as follows. If the number of pin lines in step $t$ is $Z_{p l}=1$, then the number of traces equals $N$. In this case, at $Z_{p l}=2$, the number of traces equals $2 N$. Consequently, with $Z_{p l}$ pin lines, the number of traces is as follows:

$$
Z_{n t}=Z_{p l} N
$$

At that, the distance between pin traces can be found as follows:

$$
\delta=\frac{t}{Z_{n t}}=\frac{t}{Z_{p l} N} .
$$

Considering equation (3), we obtain the following:

$$
\begin{aligned}
& \delta=\frac{c}{N}, \text { from which: } \\
& c=\delta N .
\end{aligned}
$$

Equation (6) can be used to determine the width of the $A B C$ triangle base and use that to determine the number of pin lines. At that, it is necessary to set the distance between traces $\delta$ based on technological requirements. Afterwards, one can draw right and left pin lines. Right pin lines are parallel to the $A B$ side, while left pin lines are parallel to 
the $B C$ side of triangle $A B C$. They originate from the dividing points of base $A A_{2}$ onto the $A C$ segment.

Left pin lines $(D F)$ also run through the apexes of rhombuses and have their own step $t_{1}$ and a number of additional pin lines $\mathrm{Z}_{p l 1}$. However, the length of the base of triangles $A C=c$ is identical.

From the similarity of triangles ADK and MNK, we have the following:

$$
\begin{aligned}
& \frac{A K}{A D}=\frac{M K}{M N} ; \frac{t}{l}=\frac{M K}{d} . \\
& M K=\frac{t}{N}=\delta Z_{n t} .
\end{aligned}
$$

Equation (7) takes equation (1) into consideration. If equation (5) is also taken into consideration, we obtain the following:

$$
M K=\frac{t}{N}=\delta Z_{n t}
$$

that:

Following this line of reasoning, one can conclude

$$
K A_{1}=\delta Z_{n t 1}
$$

where: $Z_{n t 1}$ is the number of traces on base $M A_{1}$ from left pin lines.

$$
\begin{aligned}
& c=\delta\left(Z_{n t}+Z_{n t 1}\right)=N \delta, \\
& N=\left(Z_{n t}+Z_{n t 1}\right) .
\end{aligned}
$$$$
\text { However, } M K+K A_{1}=M A_{1}=c \text {. Consequently: }
$$

The base of the non-isosceles triangle - the distance between pin lines - is in direct proportion to the sum of right and left pin lines, while the number of the latter - to the number of transverse pin lines.

From triangles $A A_{1} D$ and $M N K$, it is possible to obtain the following correlation:

$$
\begin{aligned}
& \frac{M K}{A A_{1}}=\frac{N K}{D K} ; \frac{\delta Z_{n t}}{t}=\frac{N K}{H} ; \\
& N K=\frac{H}{t} \delta Z_{n t} .
\end{aligned}
$$

In addition, from triangles $A_{1} K N$ and $F K D$, we have the following:

$$
\begin{aligned}
& \frac{A_{1} K}{F K}=\frac{N K}{D K} ; \frac{\delta Z_{n t 1}}{t_{1}}=\frac{N \delta Z_{n t}}{H \cdot t} . \\
& t=t \frac{Z_{n t}}{Z_{n t 1}} .
\end{aligned}
$$

From the latter equation, it follows that the step of left pin lines is in direct proportion to the correlation of left and right pin lines.

As shown above, the pins are located at the intersections of rightward and leftward helixes. Pins located successively on the rightward helix shift the encountered fertilizer granules to the right, while pins located successively on the leftward helix shift these granules to the left. Such alternate shifting and the pins' own vibration provides for an even distribution of the fertilizer across the surface of the conveyor belt. In order to ensure the alternate shifting of granules, it is necessary to determine the tilt angle of pin lines.

Assuming that the conveyor is moving in the direction $v_{t}$, consider several pins placed on the surface of the conveyor belt (Fig. 3, a). As noted in the previous study [11], pins can have different shapes - rectangular, square, rhomboid, cylindrical, etc. For theoretical studies, the pin shape is set as square.

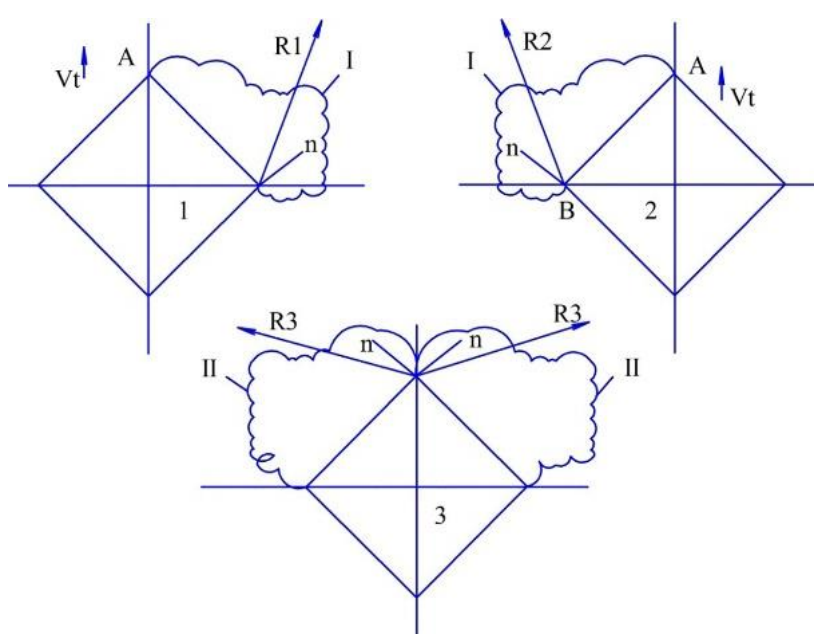

a

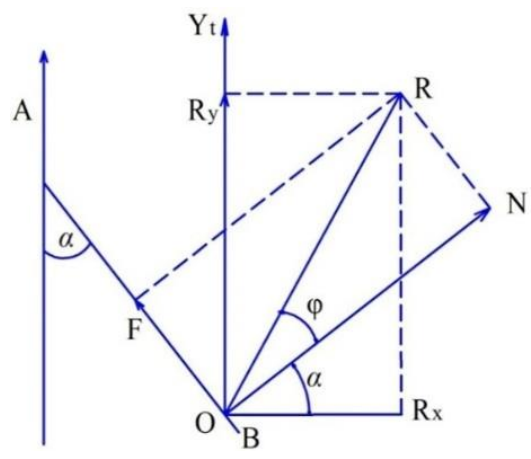

b

Fig. 3 Flowchart of transporting pin operation

The figure shows that the first and second pins exert a force on the fertilizer in the directions $R_{1}$ and $R_{2}$ and shift them towards the center, aggregate I. The third pin exerts a force in the direction $\mathrm{R}_{3}$ and moves aside the fertilizers that passed between pins 1 and 2 without impact, aggregate II. As a result of such shifting towards the center and sides, the fertilizer should be distributed evenly across the surface of the conveyor [11].

When the fertilizer is fed onto the belt surface from the bin window, the direction of speed turns by $90^{\circ}$ and its magnitude changes significantly. Therefore, the relative movement of the fertilizer at this point can be not too intensive. However, the intensity of this movement depends on 
angle $\alpha$ between the pin edge and the belt movement direction. If the $\alpha$ angle is too big, the pin works as a scraper and drags the fertilizer without relative movement. If the $\alpha$ angle is too small, the fertilizer will flow around the sides of pins and the lateral movement will be insignificant. Therefore, it is necessary to find the optimal size of angle $\alpha$ [11].

Fig. 3, b shows that edge of pin $A B$. Pint $\mathrm{O}$ of the granule that encountered the edge of the pin was affected by the following forces:

$N$ - the normal reaction of the pin surface;

$F$ - the force of friction between the pin surface and the granule;

$R$ - the resulting force than affects granule $\mathrm{O}$;

$v_{t}$ - the belt movement direction;

$\alpha$ - the angle between the pin surface and the belt movement direction.

The resulting force can be divided into directions $X$ and $Y$ :

$$
\begin{aligned}
& \overline{R_{x}}=\overline{N_{x}}+\overline{F_{x}} . \\
& \overline{R_{y}}=\overline{N_{y}}+\overline{F_{y}} .
\end{aligned}
$$

It is necessary to find the scalar values of the equation system (11) components:

$$
\begin{aligned}
& N_{x}=N \cos \alpha ; F_{x}=F \sin \alpha . \\
& N_{y}=N \operatorname{Sin} \alpha ; F_{y}=F \cos \alpha .
\end{aligned}
$$

ing:

Considering (12) from (13), we obtain the follow-

$$
\begin{aligned}
& R_{x}=N \cos \alpha-F \sin \alpha . \\
& R_{y}=N \sin \alpha-F \cos \alpha .
\end{aligned}
$$

Based on Fig. 3, one can find:

$$
\operatorname{tg}(\alpha+\varphi)+\frac{R_{y}}{R_{x}}
$$

Considering (14):

$$
\operatorname{tg}(\alpha+\varphi)=\frac{N \sin \alpha+F \cos \alpha}{N \cos \alpha-F \sin \alpha}
$$

By inserting the frictional force value $F=f N$ we obtain the following:

$$
\operatorname{tg}(\alpha+\varphi)=\frac{\sin \alpha+f \cos \alpha}{\cos \alpha-f \sin \alpha}
$$

As seen in (15), with an increase in angle $\alpha$, angle $(\alpha+\varphi)$ also increases and tends to $\frac{\pi}{2}$. In this case, the granules cease to slide along the surface of the pin. In this case, the pin works as a scraper. This happens if:

$$
\cos \alpha_{m}-f \sin \alpha_{m}=0 ; f=\operatorname{ctg} \alpha_{m} .
$$

The latter equation can be used to determine:

$$
\operatorname{tg} \varphi=\operatorname{tg}\left(\frac{\pi}{2}-\alpha_{m}\right) ; \varphi=\frac{\pi}{2}-\alpha_{m}, \alpha_{m} \leq \frac{\pi}{2}-\varphi
$$

As seen from (16), in order to ensure a relative movement of granules, the angle between the pin surface and the belt movement direction should be smaller than $\left(\frac{\pi}{2}-\varphi\right)$.

In laboratory conditions, angle $\alpha$ was set at $30^{\circ}$, $35^{\circ}, 40^{\circ}, 45^{\circ}$, and $50^{\circ}$. At that, pins at a $40^{\circ}$ angle provided for a better mixing of fertilizer granules and their even distribution across the surface of the conveyor belt.

In order to rationalize the location of the belt-pin transporter in the longitudinal plane, consider fertilizer particle $A$ in a state of equilibrium on the surface of the transporter. The angle between the belt and the horizontal is denoted as $\beta$. It is affected by gravity $Q$, frictional force $F$, and inertia force $P$. The frictional force carries the particle upwards with the belt, while the inertia force tries to roll it downwards (Fig. 4).

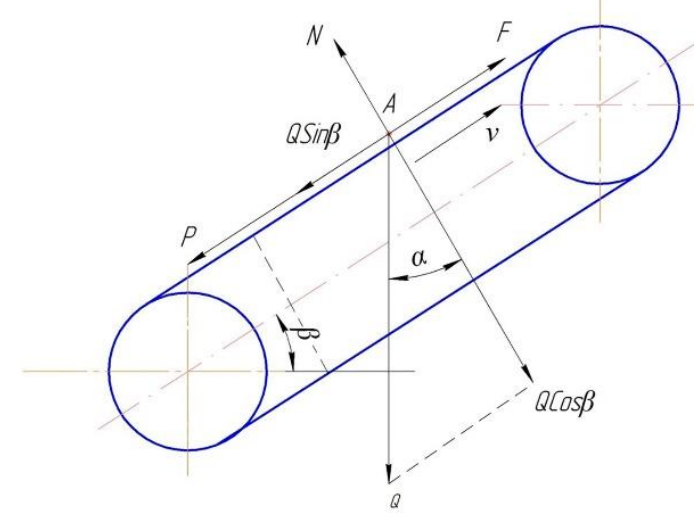

Fig. 4 To the rationalization of transporter parameters

Gravity is divided into two components: along the belt and perpendicular thereto. The equilibrium condition of particle A on the belt is as follows:

$$
F=P+Q \sin \beta
$$
is as follows:

The condition of particle movement with the belt

$$
F \geq P+Q \sin \beta
$$

where: $Q=m g ; P=m j ; m$ is the mass of the particle; $g$ is gravitational acceleration; $j$ is inertial acceleration of the particle; $F=f N, N$ is the normal reaction of the transporter surface; $f=\tan \varphi$ is the factor and tangent of the angle of friction between the particle and the transporter surface. it, we obtain:

By inserting the values into (17) and transforming

$$
m j=f Q \cos \beta-Q \sin \beta,
$$


$j=g(f \cos \beta-\sin \beta)$.

Take a designation:

$i=f \cos \beta-\sin \beta$.

At that, Eq. (18) is as follows:

$j=i g$.

Equation (20) shows that the inertia acceleration of the particle located on the tilted belt is in proportion to gravity acceleration with regard to factor $i$. The latter directly depends on the friction factor and tilt angle.

Equation (18) is transformed:

$$
\begin{aligned}
& \frac{j}{g}+\sin \beta=t \cos \beta . \\
& \frac{j}{g} \sqrt{1+\tan ^{2} \beta}+\tan \beta \leq \tan \varphi .
\end{aligned}
$$

Equation (21) shows that the tilt angle of belt $\beta$ should not exceed the friction angle. Otherwise, the particle will not move upwards with the belt.

The inertia acceleration of the particle can be expressed via velocity changes:

$$
j=\frac{v-v_{0}}{t-t_{0}},
$$

where: $v_{0}=0$ is the particle velocity at initial moment $t_{0}=0$.

In $t$ time, the particle velocity at the tailing should be equal to the speed of the loaded strand of the conveyor belt $v$. At that, the time that the particle requires to reach the speed of the transporter is as follows:

$$
t=\frac{v}{j}
$$

During $t$ time, the particle will travel a distance of:

$$
l=v t=\frac{v^{2}}{j} .
$$

Considering (18), one obtains the following:

$$
l=\frac{v^{2}}{q(f \cos \beta-\sin \beta)} .
$$

In order to reach the speed of the transporter, the particle has to travel a distance equal to $l$. Consequently, the useable length of the transporter should be as follows:

$$
l_{p} \geq l
$$

In order to rationalize the longitudinal placement of pins, consider edge $C D$ that is located at angle $\gamma$ relative to the movement speed direction $v$ (Fig. 5). Without relative movement, particle $C$ will find itself at point $\mathrm{C}_{1}$ in $t$ time. However, in the presence of relative movement, this particle will slip along the edge and move to point $D_{1}$.

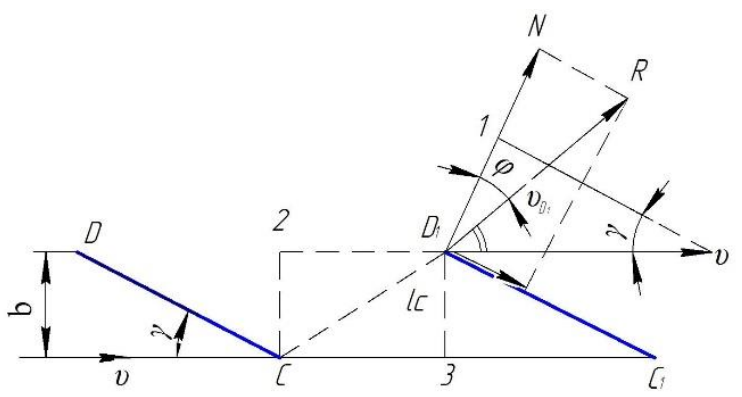

Fig. 5 To the rationalization of the longitudinal placement of pins

The particle is affected by a normal reaction $N$. Due to frictional force $F$, the resulting force $R$ deviates therefrom at angle $\gamma$. The absolute velocity of particle $C$ matches the direction of $R$.

Triangle $v D_{1} 1$ is used to determine the angle at point $D_{1}$.

$$
<v D_{1} 1=\frac{\pi}{2}-y
$$

In this case:

$$
<v D_{1} R=\frac{\pi}{2}-y-\phi
$$

By knowing this angle, one can find the distance that the particle will travel over $t$ time:

$$
\begin{aligned}
& \frac{b}{l_{c}}=\sin \left(\frac{\pi}{2}-y-\varphi\right) . \\
& l_{c}=\frac{b}{\cos (\gamma+\varphi)} .
\end{aligned}
$$

Fig. 5 shows that the path of the pin together with the transporter consists of two parts:

$$
l=C C_{1}=C 3+C_{1} 3 .
$$

Triangles $C D_{1} 3$ and $C_{1} D_{1} 3$ are used to find:

$$
C 3=l_{c} \cos \left(\frac{\pi}{2}-\gamma-\varphi\right) \text {. }
$$

Considering (19):

$$
C 3=\tan (\gamma+\varphi),
$$

$$
C_{1} 3=\frac{b}{\tan \gamma} \text {. }
$$


Insert the values into (20):

$$
l=b\left[\tan (\gamma+\varphi)+\frac{1}{\tan \gamma}\right]
$$

Formula (25) shows the minimum distance between neighboring pins in a longitudinal row, which depends on the half-width of the pin and the opening and friction angles. Smaller distance between pins may cause aggregation of the material in front of the pin edge.

Fig. 5 shows that the material that was located in the $D C D$ triangle moves in the direction of velocity $v_{a}$, slides along the edge of $D_{1} C_{1}$, and runs off of it at point $D_{1}$. The material that ran off of the $C D$ edge is replaced by the material from triangle $C D_{1} C_{1}$. The area of triangle $D C D_{1}$ depends no angle $\gamma$. It is necessary to find a value of $\gamma$, at which the area under consideration is minimum (a smaller area would not redistribute the material, since the pins have minimum impact thereon).

The weight of the material in front of edge $C D$ equals:

$$
m g=w \rho,
$$

where: $w$ is the volume of the material in front of edge $C D$; $\rho$ is the volume weight of the material. lowing:

Based on the previous equation, we have the fol-

$$
m=\frac{1}{q} w \rho .
$$

In (26), volume $w$ equals:

$$
w=\left(D D_{1} \times C 2 \times h\right) \frac{1}{2},
$$

where: $h$ is the mean thickness of material deposition in front of the pin edge:

$$
D D_{1}=C C_{1}=l \text {. }
$$

$C 2=8-$ semi-width of the pin. following:

By inserting the values into (26), one obtains the

$$
m=\frac{1}{2 q}\left[\tan (\gamma+\varphi)+\frac{1}{\tan \gamma}\right] b^{2} h \rho .
$$

In Eq. (27) $b, h, \rho$, and $\varphi$ do not depend on $\gamma$ - they are constants for this construction or these technological conditions. It is necessary to find the minimum of function $m(\gamma)$. To that end, the first derivative of the functions should be equated to zero:

$$
\frac{d m}{d \gamma}=\frac{b^{2} h p}{2 q}\left[\frac{1}{\cos ^{2}(\gamma+\varphi)}-\frac{1}{\sin ^{2} \gamma}\right]=0 .
$$

In this equation, the first member cannot be equal to zero. Consequently:

$$
\frac{1}{\cos ^{2}(\gamma+\varphi)}-\frac{1}{\sin ^{2} \gamma}=0 .
$$

This equation can be performed if:

$$
\cos (\gamma+\varphi)=\sin \gamma=\cos (90-\varphi) .
$$

From which:

$$
\begin{aligned}
& 2 \gamma=90-\varphi \\
& \gamma_{\min } \geq \frac{1}{2}\left(\frac{\pi}{2}-\varphi\right) .
\end{aligned}
$$

Correlation (28) shows that the opening angle on the frontal edge of the pin cannot be smaller than the indicated value.

\section{Results and discussion}

In order to graphically illustrate the nature of changes in the material mass exposed to the impact of transporter pins, it is necessary to calculate its dependencies on the angles of friction between the fertilizer and metal and the opening of pin $\gamma$ according to formula (27). To that end, the following data were taken from literary sources: $\rho=1.0^{2} / \mathrm{cm}^{3}$ - volume weight of the superphosphate; $\varphi=$ $25^{\circ} ; 30^{\circ} ; 35^{\circ} ; 40^{\circ}-$ angle of friction between the mineral fertilizer and metal; $\gamma=30^{\circ} ; 35^{\circ} ; 40^{\circ} ; 45^{\circ}$ - opening angle of the frontal edge of the pin; $h=1.0 \mathrm{~cm}$ - thickness of the material on the transporter: $\theta=1.0 \mathrm{~cm}$ - frontal width of the pin edge; $\varphi$ is the angle of friction between the fertilizer and metal; $\gamma$ is the angle of the frontal edge of the pin

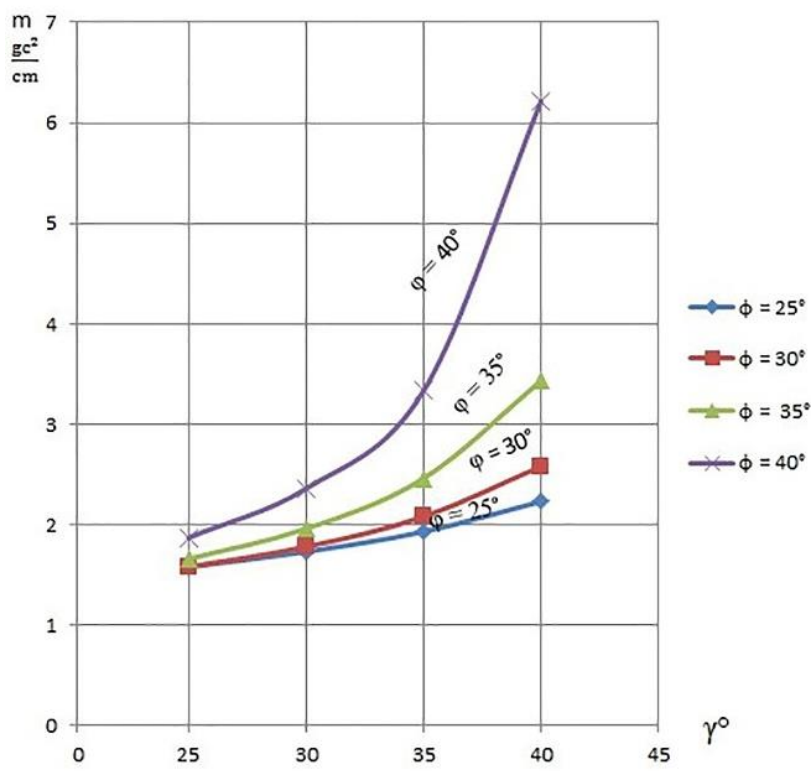

Fig. 6 Dependences of the mass of the material exposed to the pin impact on the angles of friction and opening of the edge

The calculated weights of the material that interacts with the pin are presented in the form of a diagram in Fig. 6. They show that at small angles of friction between 
the mineral fertilizer and metal, the pin opening angle affects the mass of the interacting material insignificantly. In Fig. 6, these are curves $\varphi=25^{\circ}$ and $\varphi=30^{\circ}$. At large angles of friction, the interaction between the pin and the material is significant - curves $\varphi=35^{\circ}$ and $\varphi=40^{\circ}$.

This is explained by the fact that with small friction angles, the sliding is more intensive than with large angles. It should also be noted that at small angles of the frontal edge of the pin, the sliding is more intensive than with pins with greater frontal angles, when the deceleration of the material is especially intensive at high friction levels [11].

The intensiveness of interaction between the pin and the material can also be estimated via the distance that the particle travels over the course of their contact. In formula (23), it is expressed via the semi-width of pin 6 , friction angle $\varphi$, and opening and pin $\gamma$. Their values are taken as follows: $6=0.5 ; 0.7 ; 0.9 ; 1.1 ; 1.3 \mathrm{~cm} . \gamma=25^{\circ} ; 30^{\circ} ; 35^{\circ}$; $40^{\circ} ; 45^{\circ} . \varphi=35^{\circ}$.

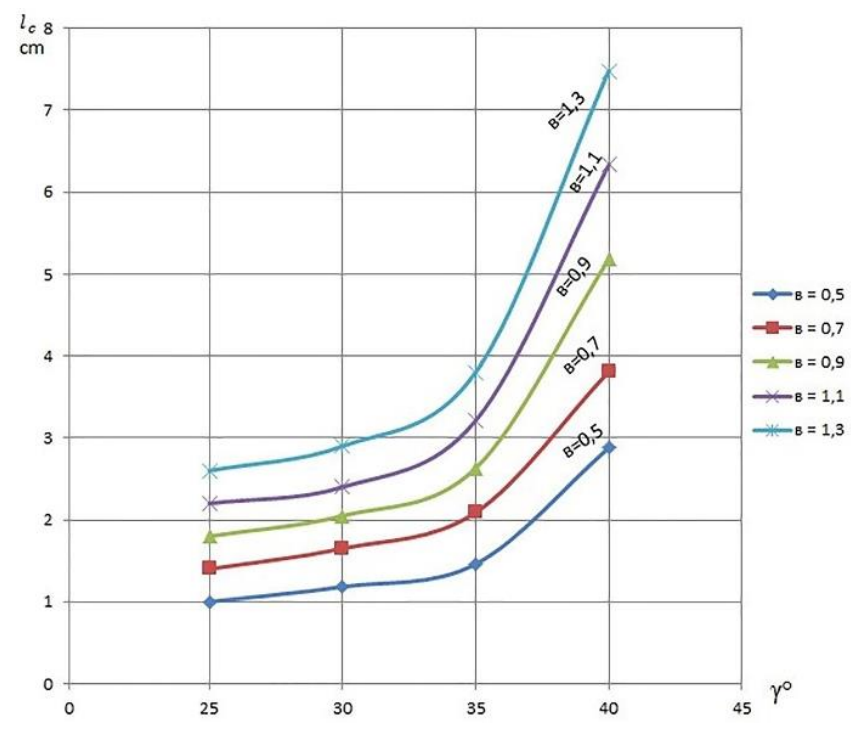

Fig. 7 Dependencies of the material particle path length on the width of the pin and the opening angle at its edge

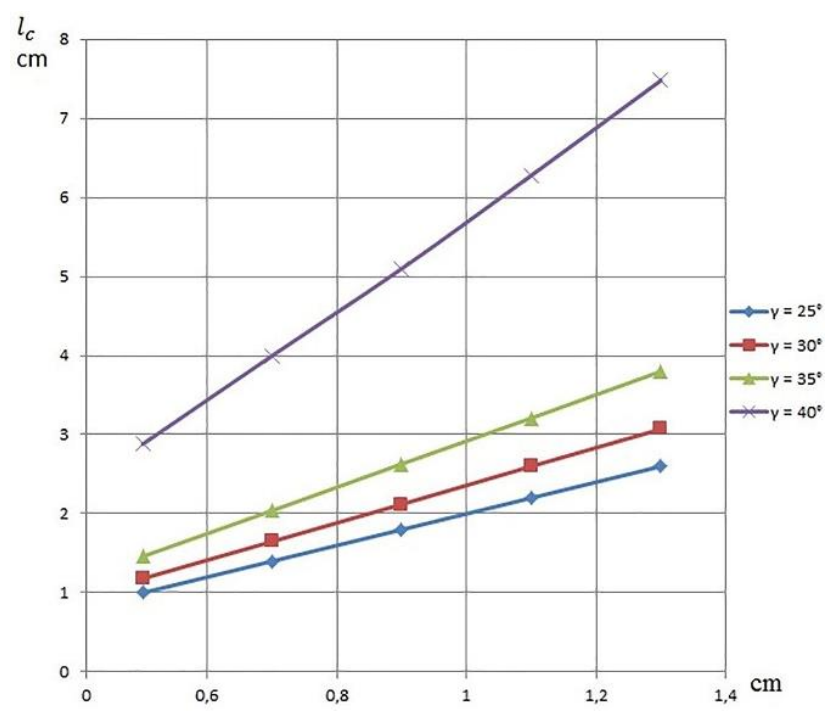

Fig. 8 Dependencies of the material particle path length on the semi-width of the pin

The estimated values of the particle path length are presented in Figs. 7 and 8. They show that the material particle path length asymptotically increases with an increase in the pin opening angle; the greater the angle, the more intensive the increase. It is necessary to take into consideration the fact that the particle path length depends on the pin opening angle in a curvilinear fashion, while the same path distance depends on the pin width in a rectilinear fashion, and, as noted above, is more intensive the greater the friction angles is (compare diagrams $\gamma=35^{\circ}$ and $\gamma=40^{\circ}$ ).

Fig. 9 shows the combined dependencies of the particle path length on the frontal angle of the pin opening and on its frontal semi-width. It shows that diagrams $l_{c}(\gamma)$ and $l_{c}(B)$ intersect at point $K$. At this point, $\gamma \approx 36^{\circ}$ and $B=0.8 \mathrm{~cm}$. Consequently, these values can be considered close to optimal for the frontal opening angle and semiwidth of the pin. However, they can be corrected experimentally with regard to specific physical, mechanical, and technological conditions.

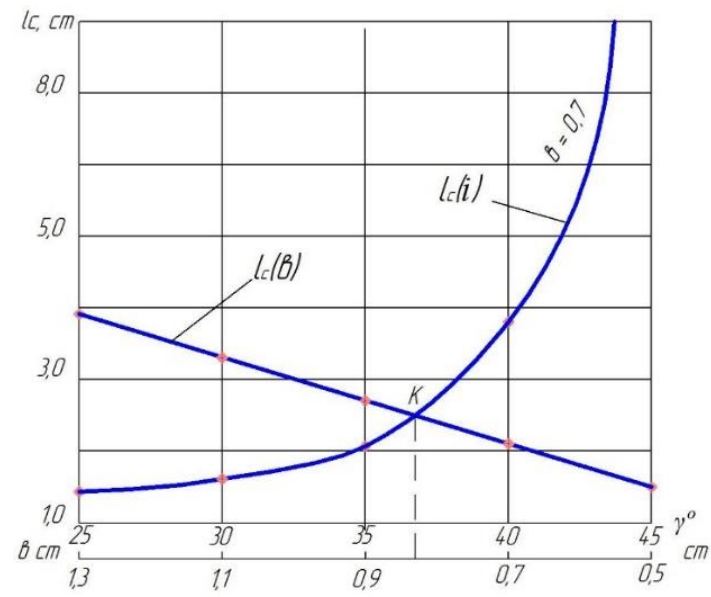

Fig. 9 Combined dependencies of the particle path length on the pin opening angle and semi-width

Belt-pin transporters with three types of pins rhombus, square, and cylinder - were manufactured for the experimental study. The height of pins was $10 \mathrm{~cm}$ and the cross section area was $100 \mathrm{~mm}^{2}$. A laboratory rig was manufactured, which allows for an infinitely variable regulation within the following ranges: transporter speed - 0.05$0.3 \mathrm{~m} / \mathrm{s}$, size of the bin flap opening $-5.0-20.0 \mathrm{~mm}$, and transporter tilt angle - 1.0-12.0 degrees. Experiments were conducted using granulated superphosphate. The experiments determined: the unevenness of sowing - deviations from the weighted average value across the row and instability of sowing - deviations from the weighted average value across the row. Deviations were assessed via coefficients of variation. Fertilizer consumption was maintained within $400 \pm 4 \mathrm{~g} / \mathrm{s}$, which corresponded to the increased introduced batch of $200 \mathrm{~kg} / \mathrm{h}$. The weighing accuracy was $0.01 \mathrm{~g}$.

After the treatment of experiment results, diagrams of dependencies of unevenness and instability on the traveling speed of the transporter, on the flap size, and on the transporter tilt angle were built. Fig. 10 shows the characteristic diagrams of changes in the fertilizer dispensing unevenness depending on the traveling speed of the transporter with rhombic pins, which showed the best results in all experiments. It shows that an increase in the transporter speed reduces unevenness; at $0.15 \mathrm{~m} / \mathrm{s}$, it stabilizes at $0.38 \%$. Then, when the speed is increased to $0.2 \mathrm{~m} / \mathrm{s}$, the unevenness starts to increase. Therefore, a transporter speed of 1.53.0 is considered optimal. 


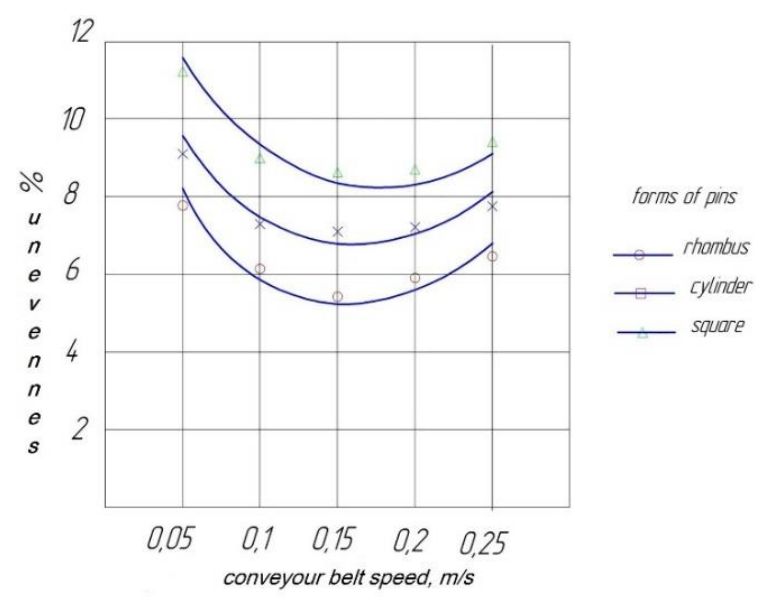

Fig. 10 Dependencies of mineral fertilizer dispensing unevenness on the conveyor belt speed

In these experiments, other factors were recorded at their optimal values $-0.14 \mathrm{~m} / \mathrm{s}$ conveyor belt speed, 14 $\mathrm{mm}$ bin flap opening, and $6^{\circ}$ transporter tilt angle; all the values were obtained from respective diagrams. It is worth noting that in instability diagrams, the nature of curves was identical to that of unevenness curves, the only difference being that their minimums differed by $5.0-7.0 \%$.

Thus, the proposed approach was tested on a pilot rig; experiments were conducted; various design options were characterized; optimal regimes and the optimal configuration of constructive parameters were determined.

Although the offered approach is developed for use in soil exposed to intensive wind erosion and hence requiring the use of gentle agricultural technologies, the approach can also be used in other types of soil, since, as mentioned above, the even distribution of fertilizers increases crop yield and the even distribution of the fertilizing material is the main advantage of the offered approach.

The offered approach to achieving an even distribution of fertilizers can also be used in other types of fertilizer dispensers.

\section{Conclusion}

A belt-pin mineral fertilizer dispenser with rhombic pins can provide for an increased fertilizer rate with 5.88.4\% dispensing unevenness and 6.1-9.3\% dispensing instability with the following technological parameters: $0.16-$ $0.18 \mathrm{~m} / \mathrm{s}$ conveyor belt speed, $13-15 \mathrm{~mm}$ bin flap opening, and 5.0-6.0 $0^{\circ}$ transporter tilt angle.

\section{Acknowledgements}

The authors would like to thank the administration of the S. Seifullin Kazakh Agrotechnical University for funding this study and services for patenting the new technological solution. The authors would also like to thank the administration of the University of Chemical Technology and Metallurgy (Sofia, Bulgaria) and D.P. Karaivanov, Sc.D. in Engineering, for the scientific and methodological consultation.

\section{References}

1. Nukeshev, S.O. 2011. Scientific bases of intra-differentiated application of mineral fertilizers in the system of precision agriculture (monograph), Astana. 358 p. (in Russian).

2. Kazantsev, N.Ya. 2004. The problem and prospects of development of mineral fertilizer application techniques in Soviet Kazakhstan in the conditions of a market economy, Grain and Grain Products 3(4): 42-43.

3. Hofstee, J.W.; Huisman, W. 1990. Handling and spreading of fertilizers part 1: Physical properties of fertilizer in relation to particle motion Review Article, Journal of Agricultural Engineering Research 47: 213234. https://doi.org/10.1016/0021-8634(90)80043-T.

4. Tola, E.; Kataoka, T.; Burce, M.; Okamoto, H.; Hata, S. 2008. Granular fertilizer application rate control system with integrated output volume measurement, Biosystems Engineering 101(4): 411-416. https://doi.org/10.1016/j.biosystemseng.2008.09.019.

5. Behbahani, A.M.; et al. 2015. Nutrient and dust enrichment in Danish wind erosion sediments for different tillage directions, EGU General Assembly 2015.

6. Krmar, M.; et al. 2015. Wind erosion on Deliblato (the largest European continental sandy terrain) studied using 210Pbex and 137Cs measurements, Journal of Radioanalytical and Nuclear Chemistry 303(3): 2511-2515. https://doi.org/10.1007/s10967-014-3841-3.

7. Vershinin, V.V.; Murasheva, A.A.; Shirokova, V.A.; Khutorova, A.O.; Shapovalov, D.A.; Tarbaev, V.A. 2016. The Solutions of the Agricultural Land Use Monitoring Problems, International Journal of Environmental and Science Education 11 (12): 5058-5069.

8. Busse, M.; et al. 2014. Innovation mechanisms in German precision farming, Precision agriculture 15(4): 403426. https://doi.org/10.1007/s11119-013-9337-2.

9. Franzen, D.W. 2011. Variability of soil test potassium in space and time, North Central Extension-Industry Soil Fertility Conference, Des Moines, IA 27.

10. Barayev, A.I. 2006. Mechanization of mineral fertilizer application during the subsurface cultivation of soil, Alma-Ata: Kaynar, 1984. 21 p. Dedicated to the 50th Anniversary of the Scientific and Production Center for Grain Farming, Ministry of Agriculture of the Republic of Kazakhstan, Shortandy: 257-264 p.

11. Filonov, V.M. 2006. The role of fertilizers in agricultural intensification. Modern Problems of Soil-Protective Farming and Ways of Improving the Stability of Grain Production in Steppe Regions: Collection of Reports from the International Scientific and Practical Conference Dedicated to the 50th Anniversary of the A.I. Barayev Scientific and Production Center for Grain Farming, Ministry of Agriculture of the Republic of Kazakhstan, Shortandy. 257-264 p.

12. Nukeshev, S.; Eskhozhin, Dz.; Lichman, G.; Karaivanov, D.; Zolotukhin, E.; Syzdykov, D. 2016. Theoretical substantiation of the design of a seeding device for differentiated intra soil application of mineral fertilizers, Actauniversitatisagriculturae et silviculturaemendelianaebrunensis 64(12,1). http://dx.doi.org/10.11118/actaun201664010115.

13. Gamzikov, G.P.; Lapukhin, T.P. 2005. A comprehensive assessment of fertilizer effectiveness with longterm consistent application, Proceedings of the 8th International Scientific and Practical Conference. Barnaul, Novosibirsk 1: 148. 
14. Shilo, I.N.; Romanyuk, N.N.; Ageychik, V.A.; Nukeshev, S.O.; Eskhozhin, D.Z.; Akhmetov, Ye.S.; Zhaksylykova, Z.S. 2013. Dispenser: Republic of Belarus patent 9706, MPK A 01S 15/00, applicant - Belarussian State Agrarian Technical University, No.u20130387; applied on 06.05.2013; published on 30.12.2013.

15. Rozmann, S. 1936. Harrows - the only geometric method of construction, Russian Institute of Agriculture and Experimental Mechanical Engineering, Theory of Construction and Manufacturing of Agricultural Machinery 1 .
D. Eskhozhin, S. Nukeshev, Z. Zhaksylykova,

K. Eskhozhin, A. Balabekova

\section{DESIGN AND STUDY OF A DISPENSER FOR THE INTRODUCTION OF THE MAIN BATCH OF MINERAL FERTILIZERS}

S u m m a r y

The analytical and experimental work designed and manufactured a belt-pin dispenser for the introduction of mineral fertilizers. The offered machine was developed for the special conditions of Central and Northern Kazakhstan soil, where general soil exposure to wind erosion leads to a wide use of subsurface soil cultivation, which causes difficulties with deep and even introduction of mineral fertilizers. The offered belt-pit dispenser for the introduction of mineral fertilizers can increase the batch of fertilizers in a wide range. The lack of portion feed provides for an even distribution of the fertilizer in the soil. The even distribution of the fertilizers on the conveyor belt and in the soil depends on the pattern of pin location on the belt surface. The study rationalized the optimal distribution of pins on the conveyor belt, its optimal pitch, and parameters of longitudinal placement of pins.

Keywords: introduction of mineral fertilizers, conveyor belt, dispenser, batching, pin line.

Received March 09, 2017

Accepted June 14, 2018 